\title{
“Segues" in Botanica: una
documentazione fotografica
}

Research

\begin{abstract}
Nel contesto di una esercitazione in classe, è stato chiesto agli studenti iscritti al corso "Introductory Ethnobotany" dell'University of Hawai $i$ at Manoa di dare un nome a 40 esemplari di piante fresche evitando di utilizzare nomi comuni. Uno degli scopi di questo esercizio è stato quello di raccogliere informazioni riguardanti la reazione visiva degli studenti. Gli studenti hanno inventato nomi correlati a forma, colore ed altre caratteristiche morfologiche delle piante campione. Un modo significativo ed esplicativo per comprendere i risultati raccolti con questo esperimento è quello di raffigurare quello che effettivamente gli studenti hanno fatto in classe. Poiché le immagini sono uno strumento potente per esprimere informazioni, il metodo utilizzato in questo esperimento è presentato qui come documento fotografico con la speranza che ricercatori e insegnanti di altre parti del mondo possano condurre simili esercitazioni in classe con i propri studenti.
\end{abstract}

\section{Introduzione}

E' stato svolto un esercizio didattico con lo scopo di introdurre la tassonomia botanica e il concetto di tecniche di consenso. Questo è stato condotto come parte dei "Segues" di Botanica (Lau et al. 2009, Reedy in revisione) nell'ambito del corso "Introductory Ethnobotany" all'University of Hawai'i at Manoa durante l'autunno 2008. La grande maggioranza degli studenti sono "major" non di orientamento scientifico. Come negli anni precedenti, uno degli scopi principali dei "Segues" di Botanica è quello di presentare a studenti "major" la botanica in modi con cui possono facilmente relazionarsi e di conseguenza apprezzare maggiormente la materia. Nondimeno, siamo stati in grado di raccogliere dati sufficienti con questo semplice esercizio didattico per capire come studenti universitari danno un nome e categorizzano le piante nel contesto di un laboratorio. I dati raccolti nel 2007 sono stati già analizzati e presentati in un articolo scientifico (Lau et al. 2009).
Poichè in Lau et al. (2009) ricercatori di altre parti del mondo sono stati incoraggiati a condurre un esperimento simile ed a condividerne i risultati nella speranza di individuare tendenze emergenti nel modo in cui gli studenti di differenti backgrounds politici e culturali, pensiamo che questa documentazione fotografica possa fornire una più chiara spiegazione di come questo esperimento sia stato condotto. In altre parole, lo scopo di questa documentazione fotografica è quello di complementare quella pubblicazione presentando i metodi utilizzati in un modo alternativo.

\section{Esercitazione in classe}

Per l'esercizio in classe sono stati mostrati quaranta campioni vegetali agli studenti, divisi in gruppi da tre a cinque, i quali hanno assegnato, a ciascuna delle piante, un nome di propria scelta. Ogni gruppo ha poi discusso e concordato tramite consenso il nome da assegnare, evitando di utilizzare nomi già noti delle piante. Inoltre, gli studenti hanno dovuto organizzare le piante in categorie utilizzando un loro proprio sistema di classificazione

\section{Correspondence}

Valentina Savo, Biology Department, Roma Tre University, Viale Marconi 446, (00146), Rome, ITALY.

vsavo@uniroma3.it

Y. Han Lau, W.C. McClatchey, D. Reedy, A.K. Chock, K.W. Bridges \& Z. Ritchey, Department of Botany, University of Hawai i at Manoa, 3190 Maile Way, 101, Honolulu, Hawai i, U.S.A.

Ethnobotany Research \& Applications 7:135-146 (2009)

Published: May 15, 2009 
usando un sistema standard di categorizzazione mediante cartoncini appositi (Bernard 2000).

Questa è una semplice esercitazione di classe il cui valore non è tanto relativo a quanto corretti possano essere i sistemi di classificazione creati dagli studenti, quanto piuttosto il sistema di nomenclatura e tassonomia in sé. Da un punto di vista scientifico, inoltre, ci si chiede se gli studenti hawaiani, che hanno diversi background culturali e di conoscenze, possano esibire simili pattern nel processo di dare un nome alle piante e classificarle. A più vasta prospettiva, ci si chiede inoltre quanto possano essere simili o differenti gli studenti hawaiani da altri in Nord e Sud America, Africa, Asia ed Europa. In linea di principio, questo non è molto diverso da come le persone di differenti culture possano dare un nome o categorizzare le piante nella loro vita quotidiana. Quindi, viene qui presentata una documentazione fotografica di questa semplice esercitazione con la speranza che possa invogliare altri ricercatori di altre parti del mondo a condurre un simile esercizio in classe con i loro studenti.

\section{La documentazione fotografica}

Le immagini fotografiche posso servire come strumento per assistere altri scienziati e ricercatori nel capire tutti i passaggi nello svolgimento di un esperimento (Vogl-Lukasser \& Vogl 2005). A tal proposito, è stata preparata una sequenza fotografica per illustrare tutti i passaggi chiave di questo esercizio. Queste fotografie mostrano il processo completo di svolgimento dell'esercizio che potrebbe essere molto utile ad altri scienziati ed insegnanti che$$
\text { a }
$$

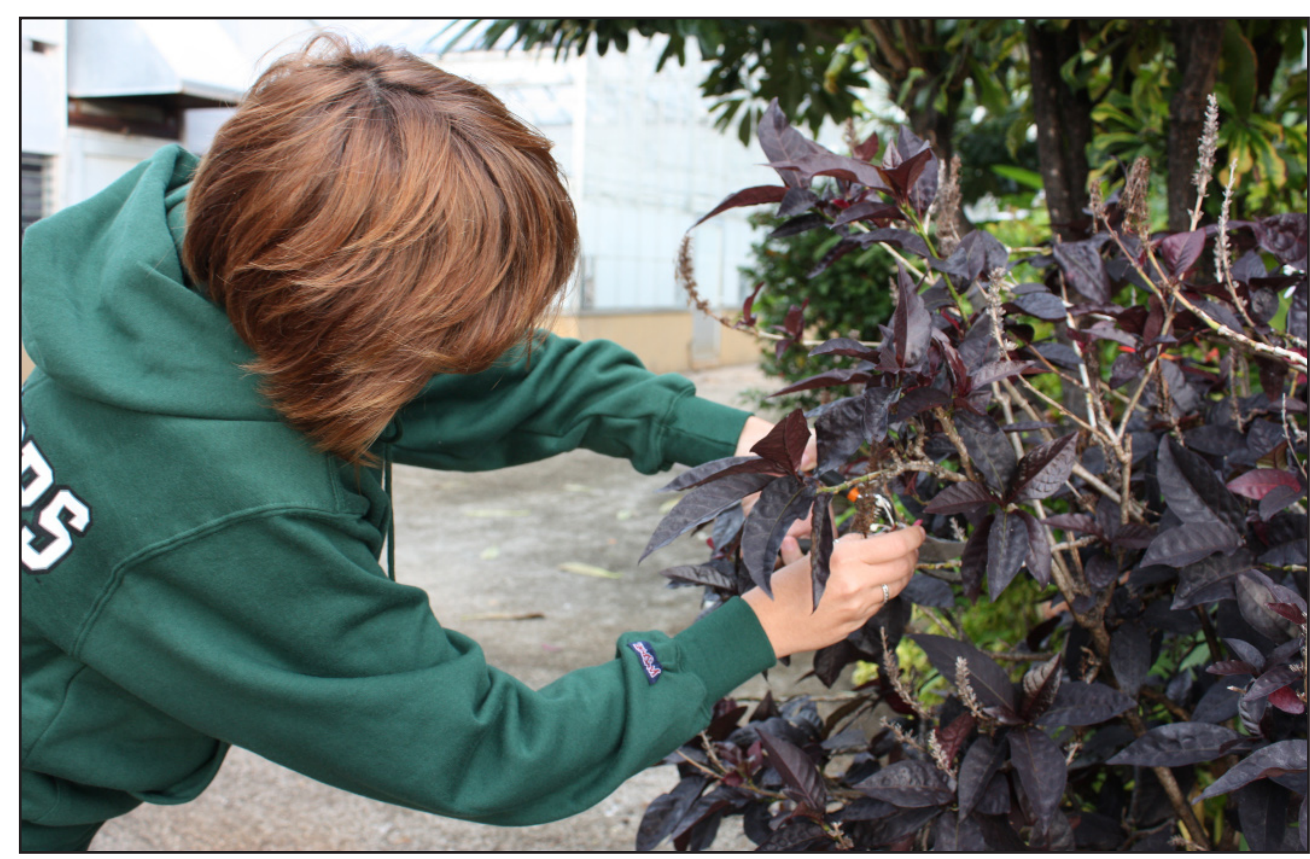

Figura 1. I campioni sono stati raccolti freschi poco prima dell'esercitazione. vogliano condurre esperimenti similari in futuro. E' stato inoltre evidenziato che le immagini fotografiche sono particolarmente utili poiché possono trasmettere informazioni complesse e, nel caso di una documentazione fotografi$\mathrm{ca}$, le foto non sono disgiunte dal contesto di ricerca (McClatchey et al. 2005).

Il consenso informato è stato richiesto ed ottenuto da tutti gli studenti che hanno partecipato a questo esercizio. Seppure siano state scattate delle fotografie anche nel 2007 , nessuna di quelle foto è stata inclusa in questo lavoro in quanto non si era provveduto ad ottenerne esplicito consenso per la pubblicazione. Tutte le foto sono state scattate usando una macchina fotografica Canon EOS Rebel XSi con una lente da 18-55 mm. Le foto qui presentate sono state scelte in modo da garantire la spiegazione più esplicativa e logica dell'esercizio con un minimo testo collegato. Le immagini non sono state sostanzialmente modificate ad eccezione di eventuali ritagli o ridimensionamenti.

Molti dei campioni vegetali sono stati raccolti da piante coltivate nelle vicinanze del campus universitario (Figura 1) in modo da assicurare che questi fossero freschi. Alcuni frutti e ortaggi (ad esempio la melanzana e la patata) sono stati invece acquistati da supermarket in zona. Tutto il materiale vegetale, ad esclusione di frutti e ortaggi, è stato posto in barattoli riempiti con acqua in modo da evitarne la disidratazione. Un numero è stato inoltre associato ad ogni campione vegetale.

L'esercitazione di classe è stata condotta in uno dei laboratori dell'Università (Figura 2). Gli studenti sono stati esposti ad un ambiente colorato con immagini di piante e strutture vegetali sulle pareti (Figura 3) che hanno contribuito come stimoli addizionali per gli studenti. II contesto della classe scelta è importante poiché è stato evidenziato che un ambiente positivo può a sua volta avere effetti positivi nella risoluzione di problemi (Sinclair et al. 1998).

Le piante sono state collocate nella stessa identica disposizione utilizzata nell'esperimento svolto nel semestre autun- 


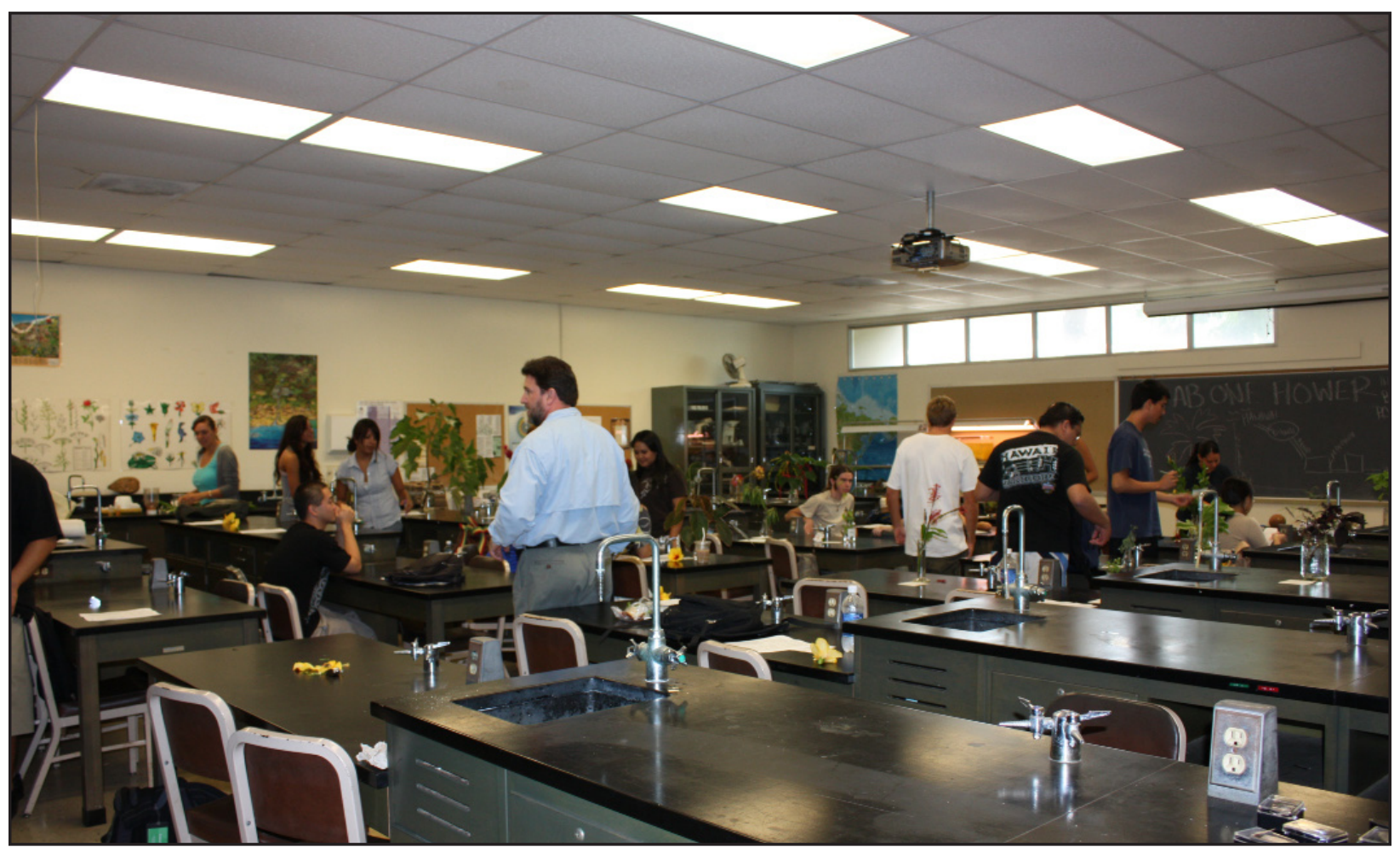

Figura 2. La classe dove è stata condotta l'esercitazione.

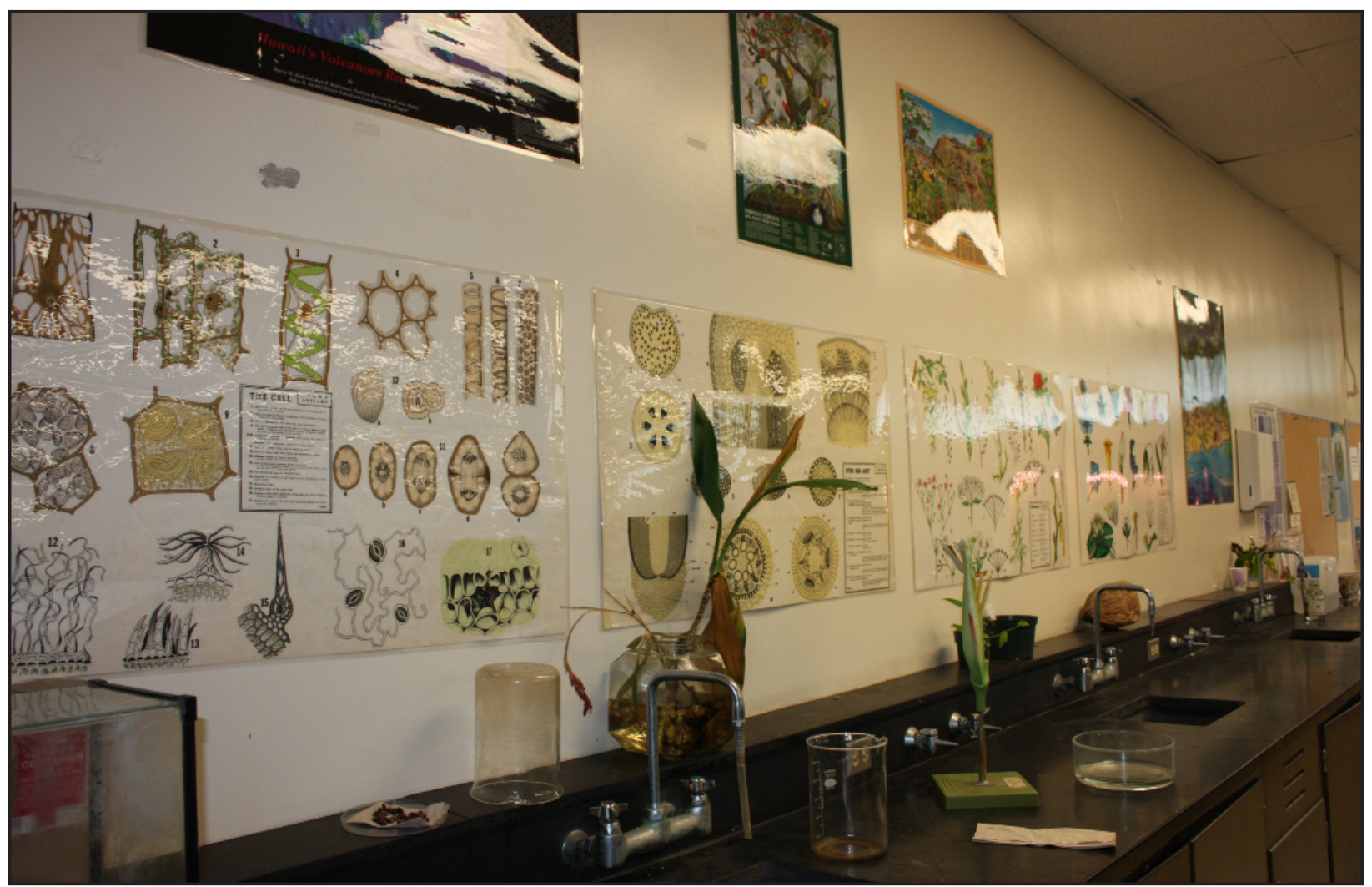

Figura 3. Sulle pareti del laboratorio sono esposti dei poster con informazioni relative alla botanica. 
nale del 2007 (Figura 4). Questo è importante per mantenere un certo livello di consistenza tra gli esperimenti svolti nei due semestri.

In generale, la nomenclatura popolare (tradizionale) delle piante è principalmente basata sulla morfologia, ecologia (habitat) e funzione (Berlin et al. 1966, Berlin 1973, Ceuterick \& Van Damme 2006). Pertanto, nell'esercizio sono stati utilizzati diversi tipi di parti di piante come rami, foglie, fiori, frutti e radici. Inoltre, i campioni vegetali presentavano diversi tipi di caratteristiche come profumi, colori e forme (Figura 5) che erano caratteristiche distintive che gli studenti hanno potuto facilmente percepire. Gli studenti infine hanno potuto toccare (Figure 6 e 7) o annusare le piante (Figura 8) mentre decidevano quale nome utilizzare (Figure 9 e 10). Comunque, siccome l'esperimento è stato svolto in un ambiente chiuso, agli studenti sono state precluse informazioni ecologiche come ad esempio il portamento delle diverse specie.

Nella seconda parte dell'esercizio è stato chiesto agli studenti di organizzare i nomi associati alle piante in approssimativamente da 3 a 6 categorie in base a similarità e differenze (Figure da 11 a 13). Il processo di categorizzazione è stato forse quello più impegnativo di tutta l'esercitazione e sono stati osservati diversi comportamenti sociali durante questa parte. Alcuni gruppi hanno mostrato una certa cooperazione egualitaria tra i membri, mentre altri hanno evidenziato chiaramente un certo tipo di comportamenti di leadership emergenti nel gruppo (Figure 14 e 15). Nonostante ciò, è risultato chiaro che questi differenti pattern sociali non hanno significativamente influito sul fatto che siano state usate frequentemente la combinazione sostantivo-aggettivo per dare un nome alle piante e i sostantivi da soli per dare un nome alle categorie (Lau et al. 2009). Questo processo di dare un nome può essere potenzialmente comparato con l'accettazione sociale di un neologismo che viene inizialmente utilizzato da un individuo (o piccolo gruppo) e viene poi diffusamente accettato anche se i due processi possono non essere esattamente la stessa cosa. Dopo che gli studenti hanno completato il processo di categorizzazione delle piante (Figura 16), tutti i dati sono stati organizzati ed analizzati per identificare i modi in cui gli studenti nominano e categorizzano le piante. Infine, tutte le piante sono state preparate come campioni d'erbario (Figura 17) e depositate all'University of Hawai'i Herbarium (HAW).

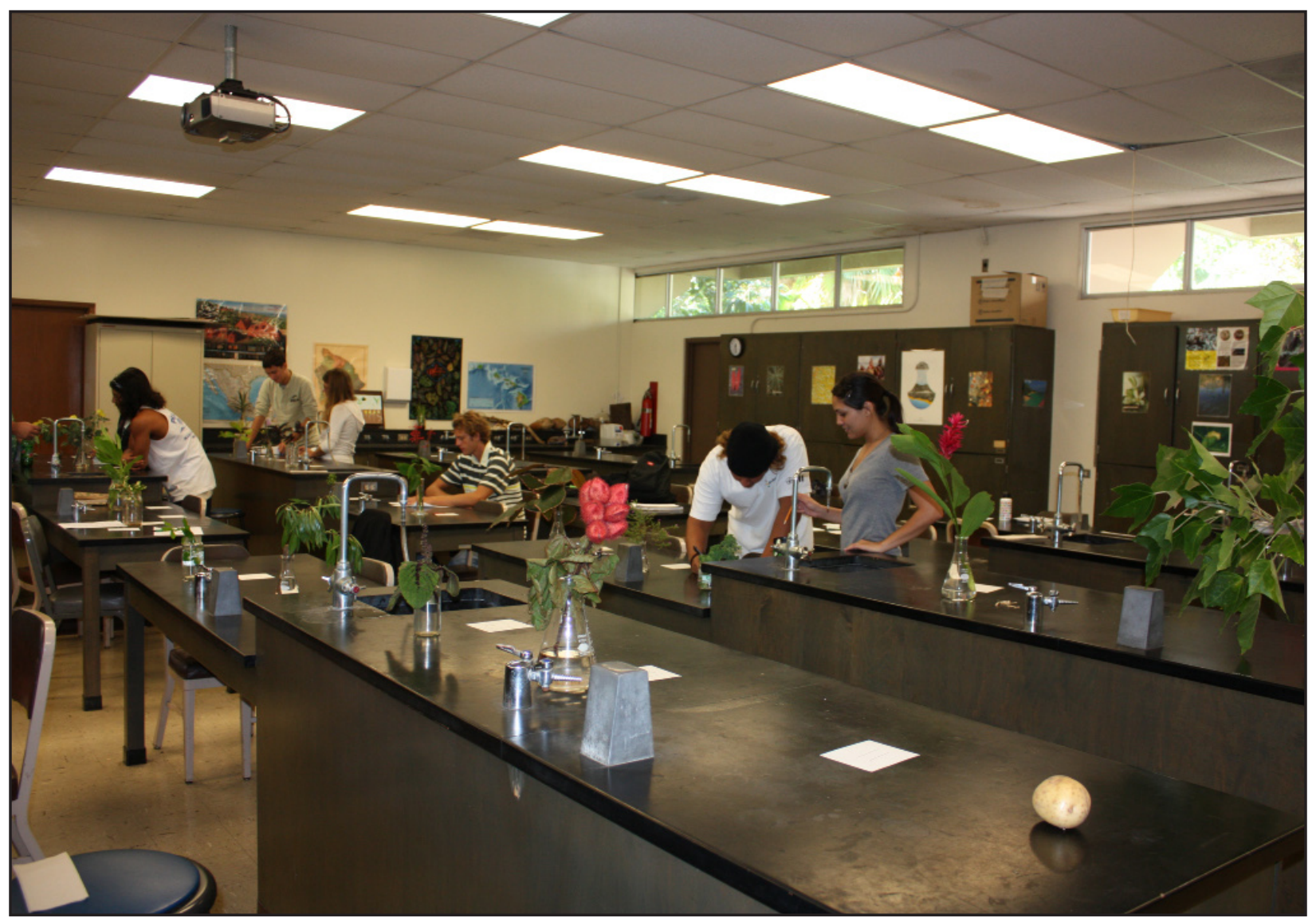

Figura 4. Le piante utilizzate sono le stesse impiegate durante l'esperimento condotto durante il semestre autunnale del 2007 e sono state posizionate nello stesso ordine. 


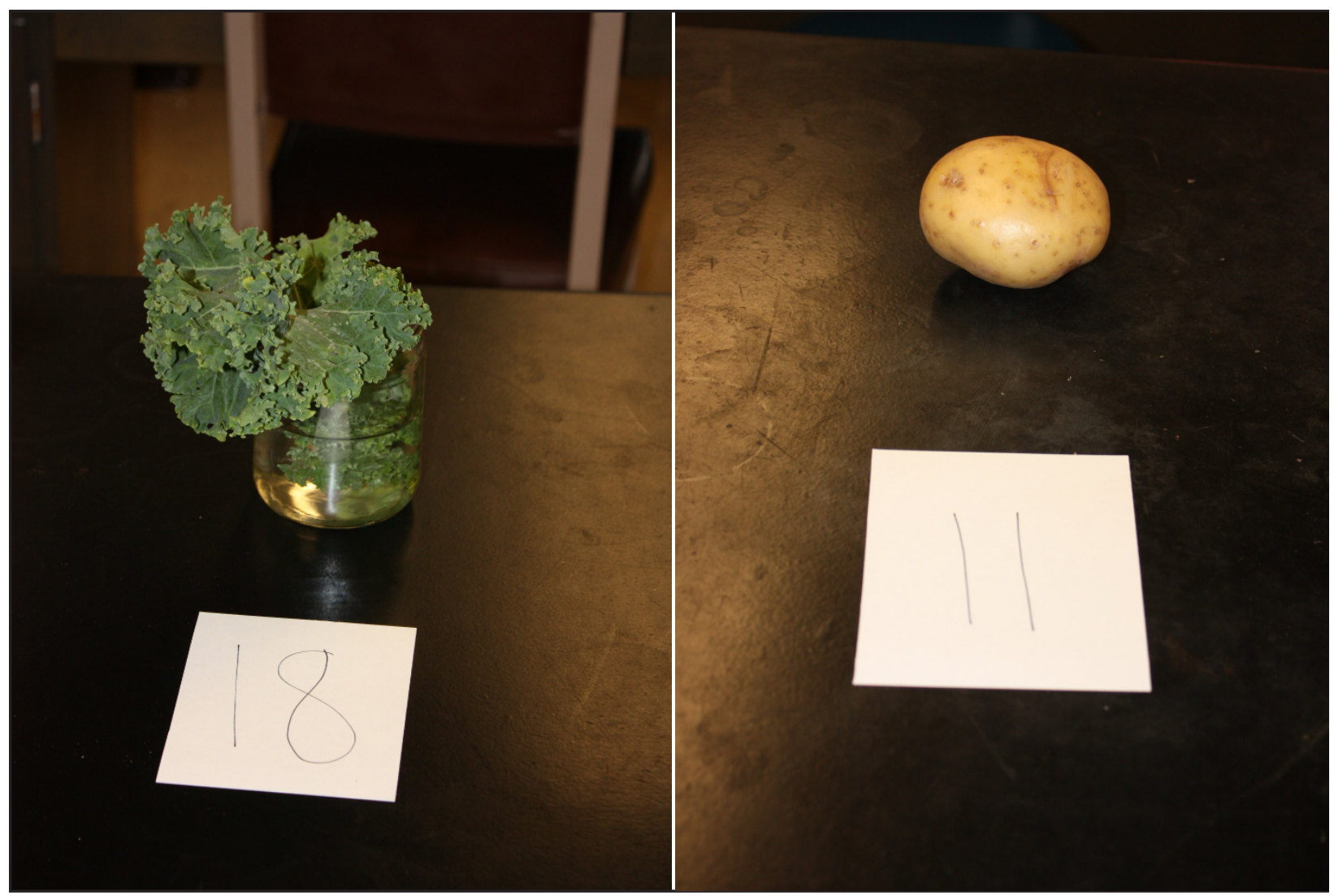

Figura 5. Un diversificato range di caratteri come ad esempio colori e forme, sono serviti agli studenti come stimoli.

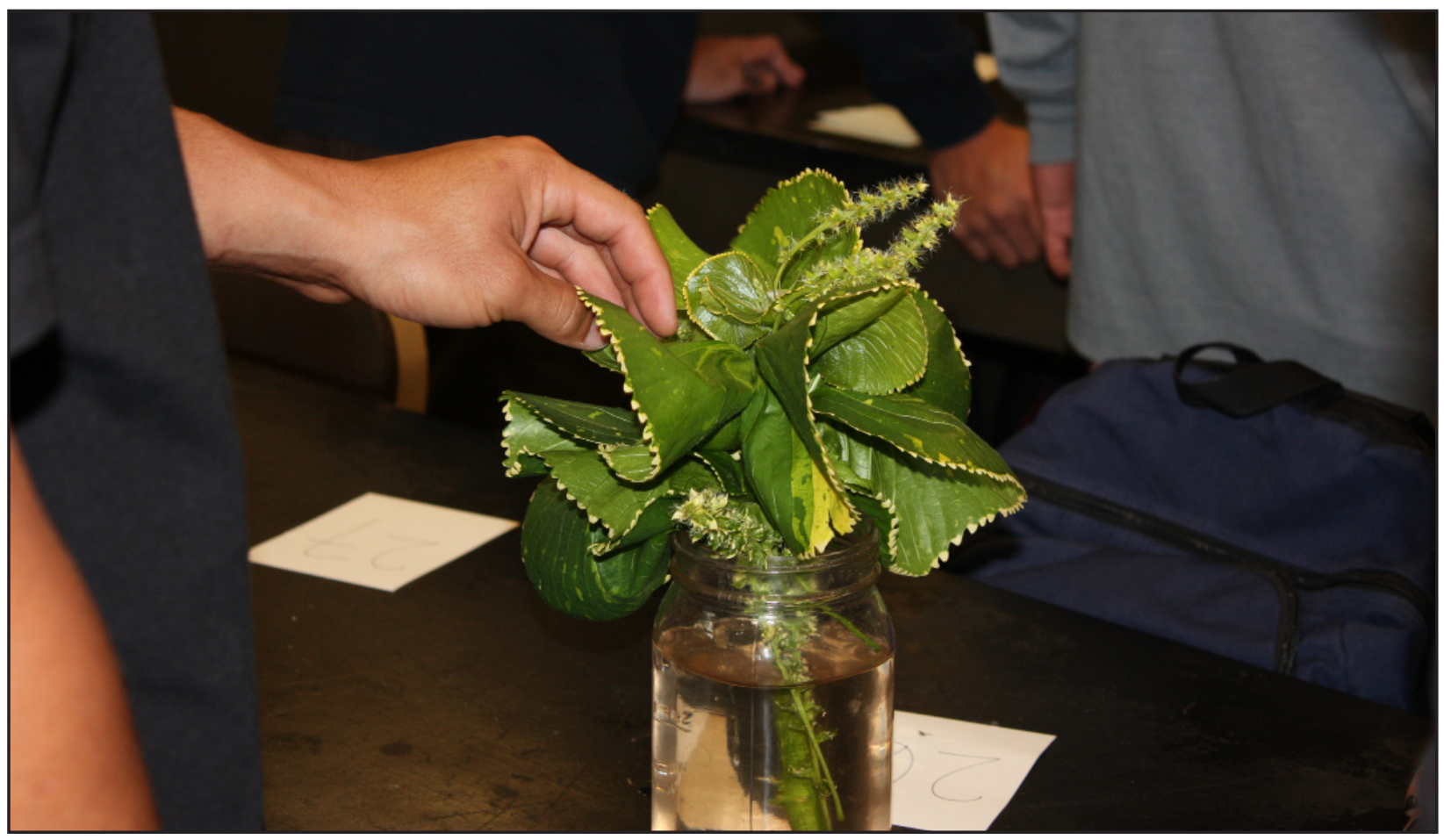

Figura 6. Uno studente sta testando la consistenza della foglia di un campione. 


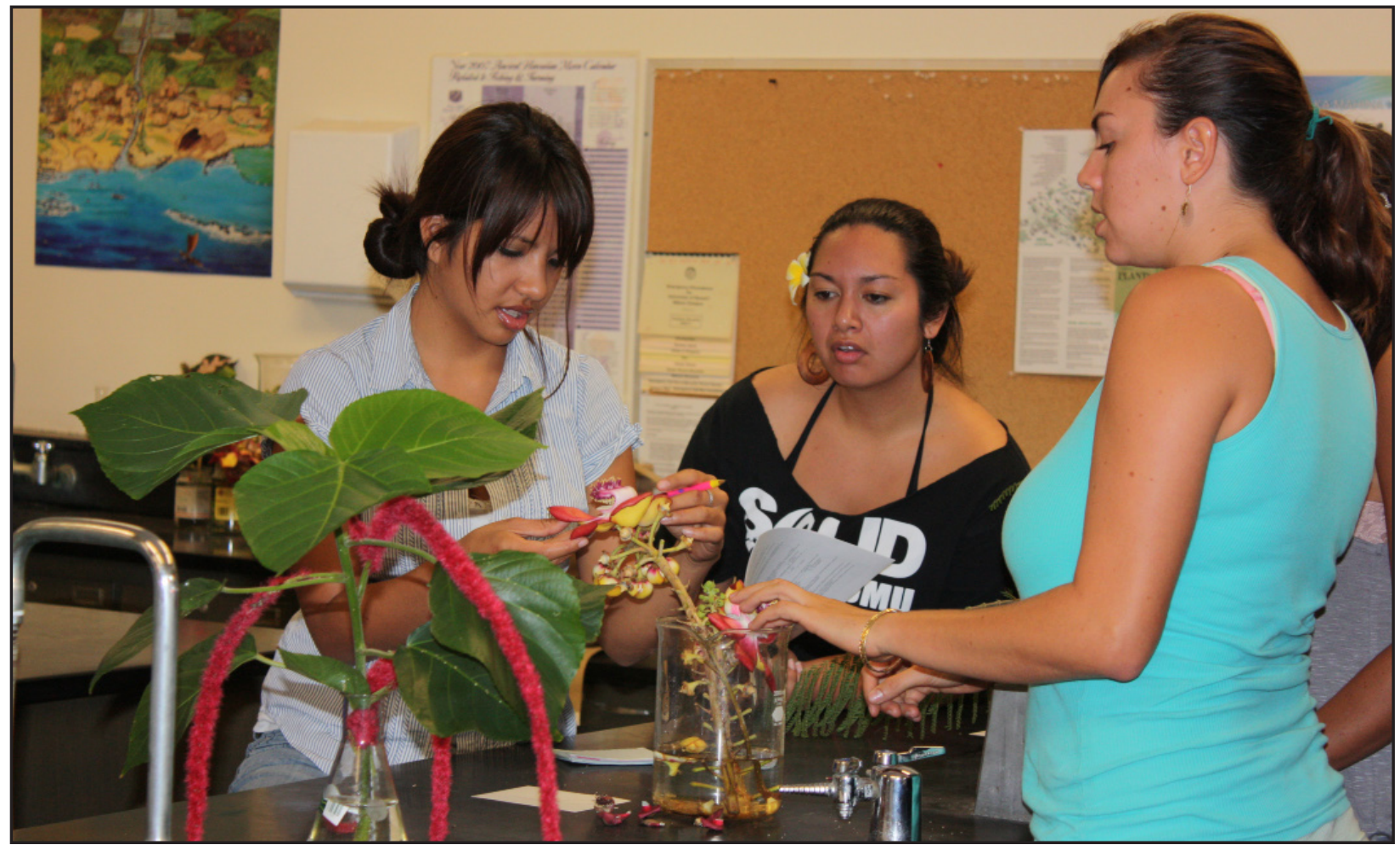

Figura 7. Una studentessa sta esaminando il fiore di una pianta mentre le sue partner di gruppo la osservano.

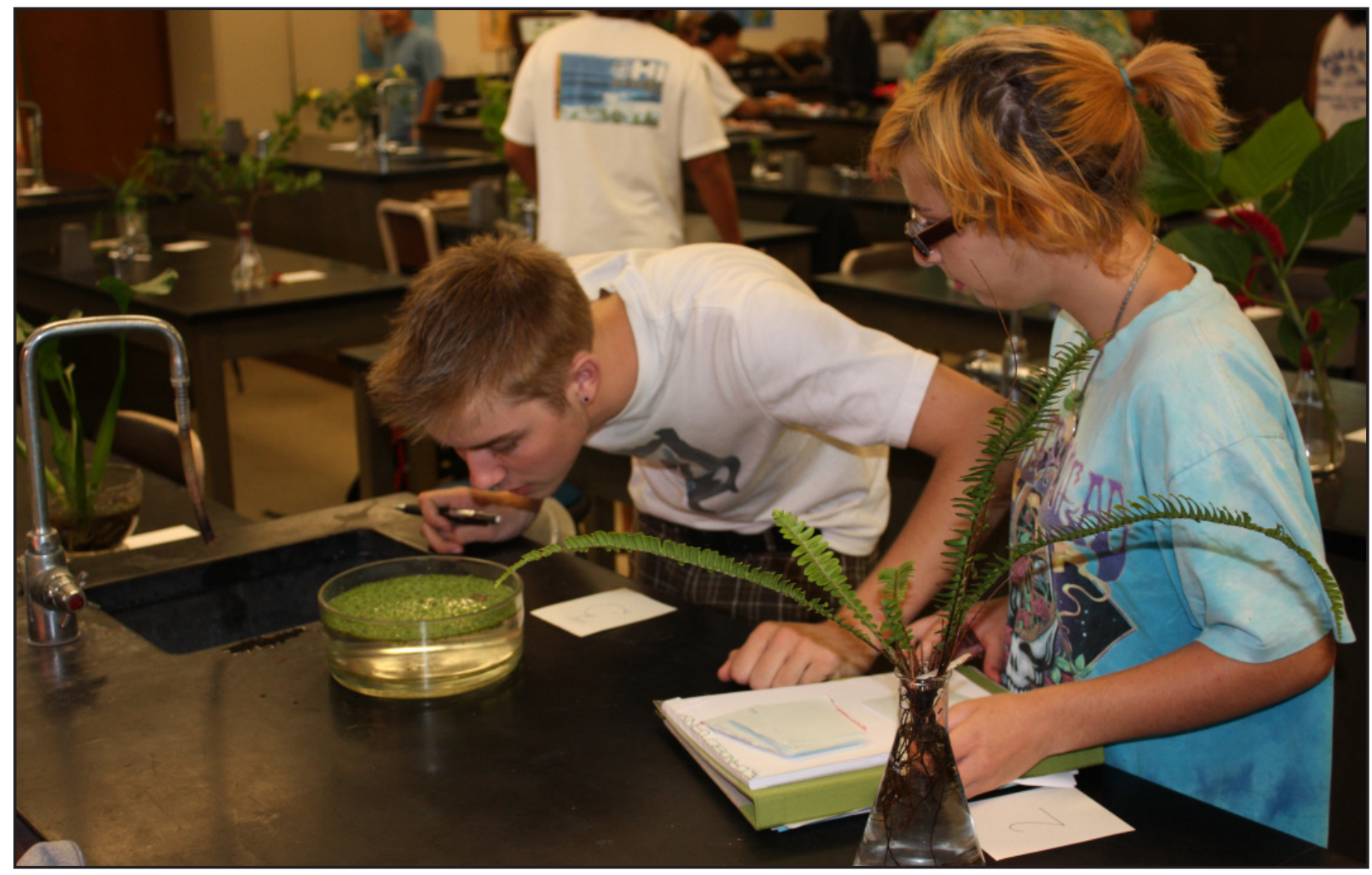

Figura 8. Uno studente annusa un recipiente contenente un campione di piante acquatiche mentre una partner di esercizio osserva. 


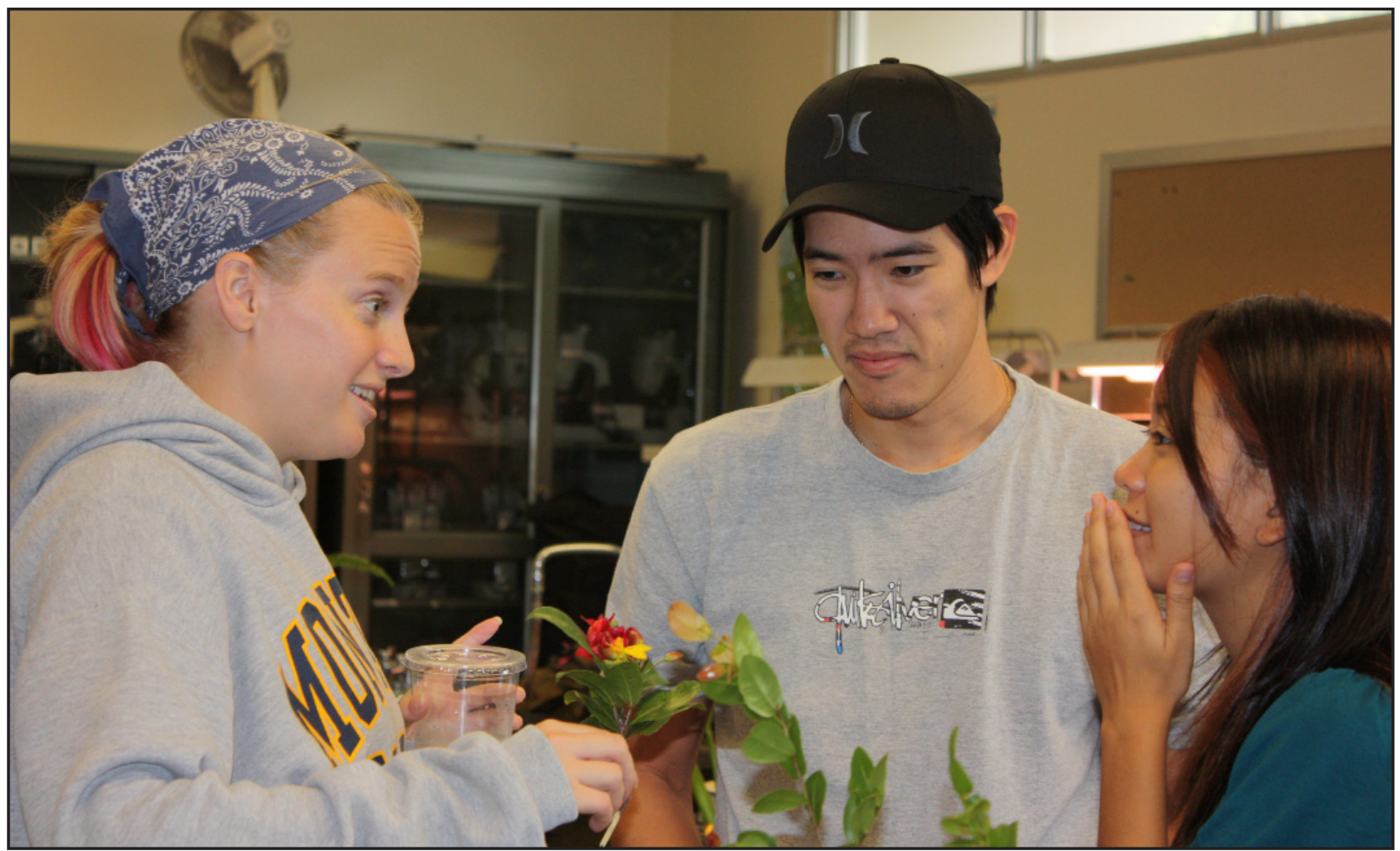

Figura 9. Un gruppo entusiasta di studenti sta discutendo e decidendo il nome per un campione vegetale.

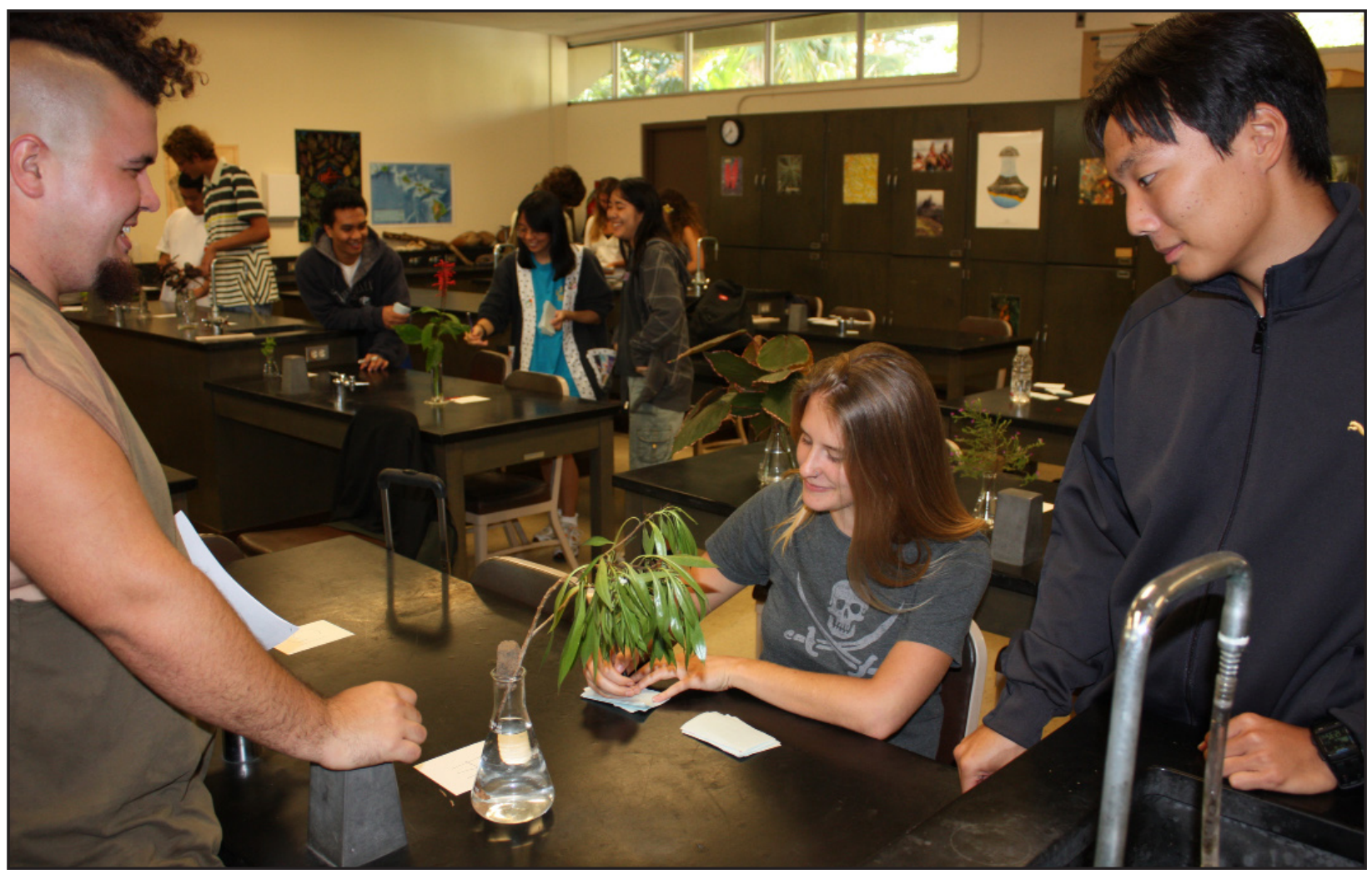

Figura 10. Gli studenti si divertono dando i nomi alle piante. 


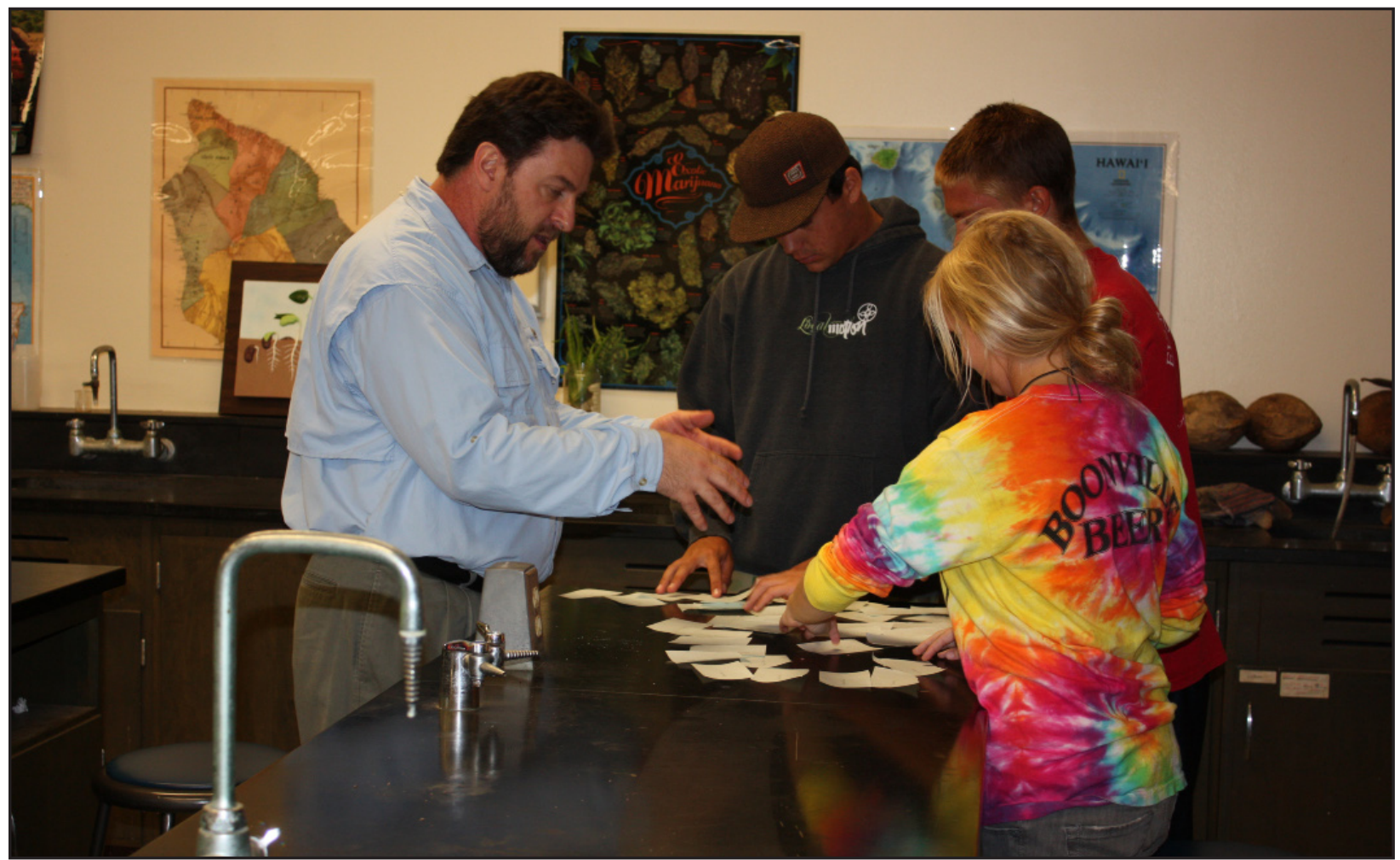

Figura 11. II docente del corso sta spiegando l'esercizio di classificazione ad un gruppo di studenti.

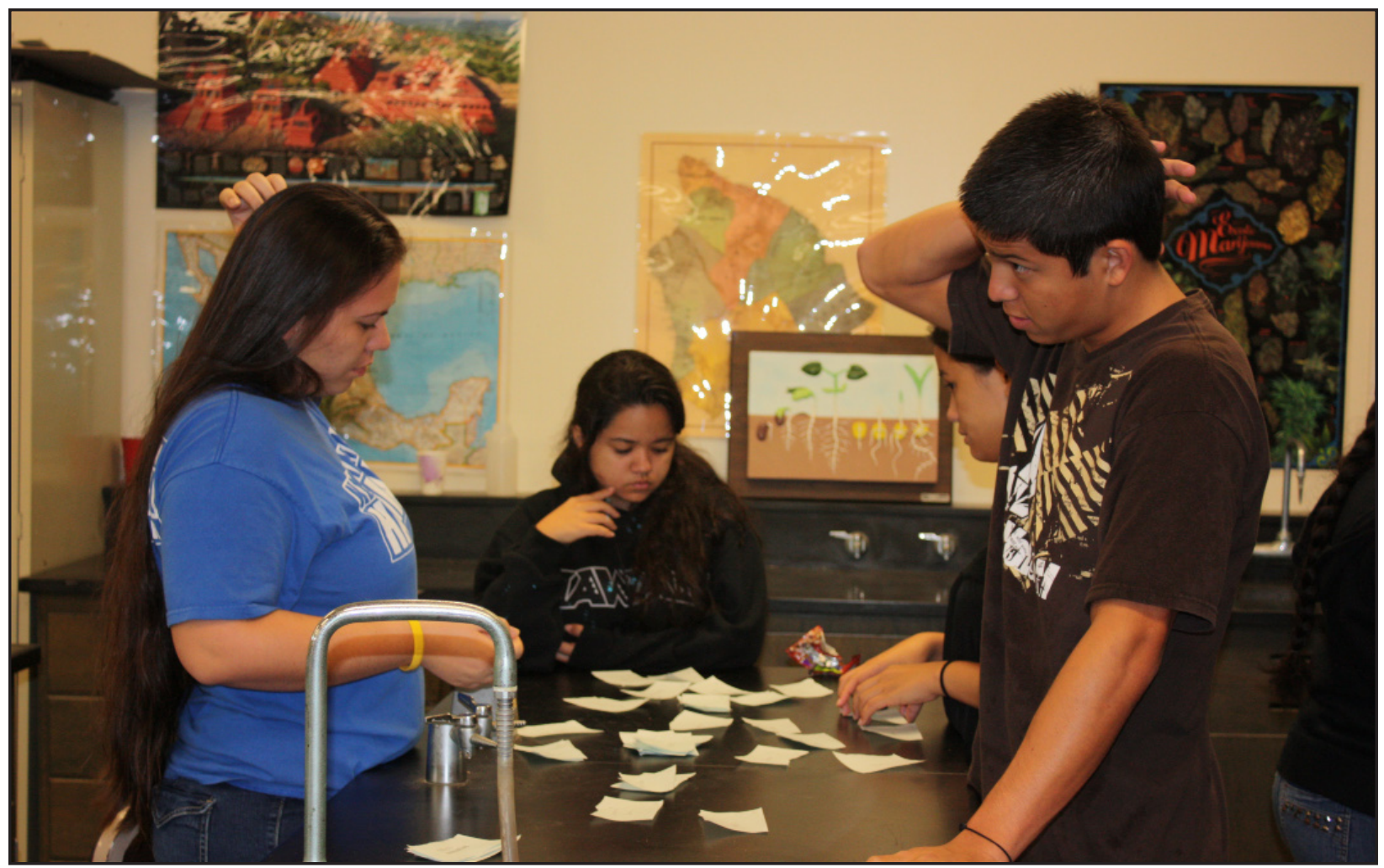

Figura 12. Gli studenti categorizzano le piante in base a similarità e differenze. 


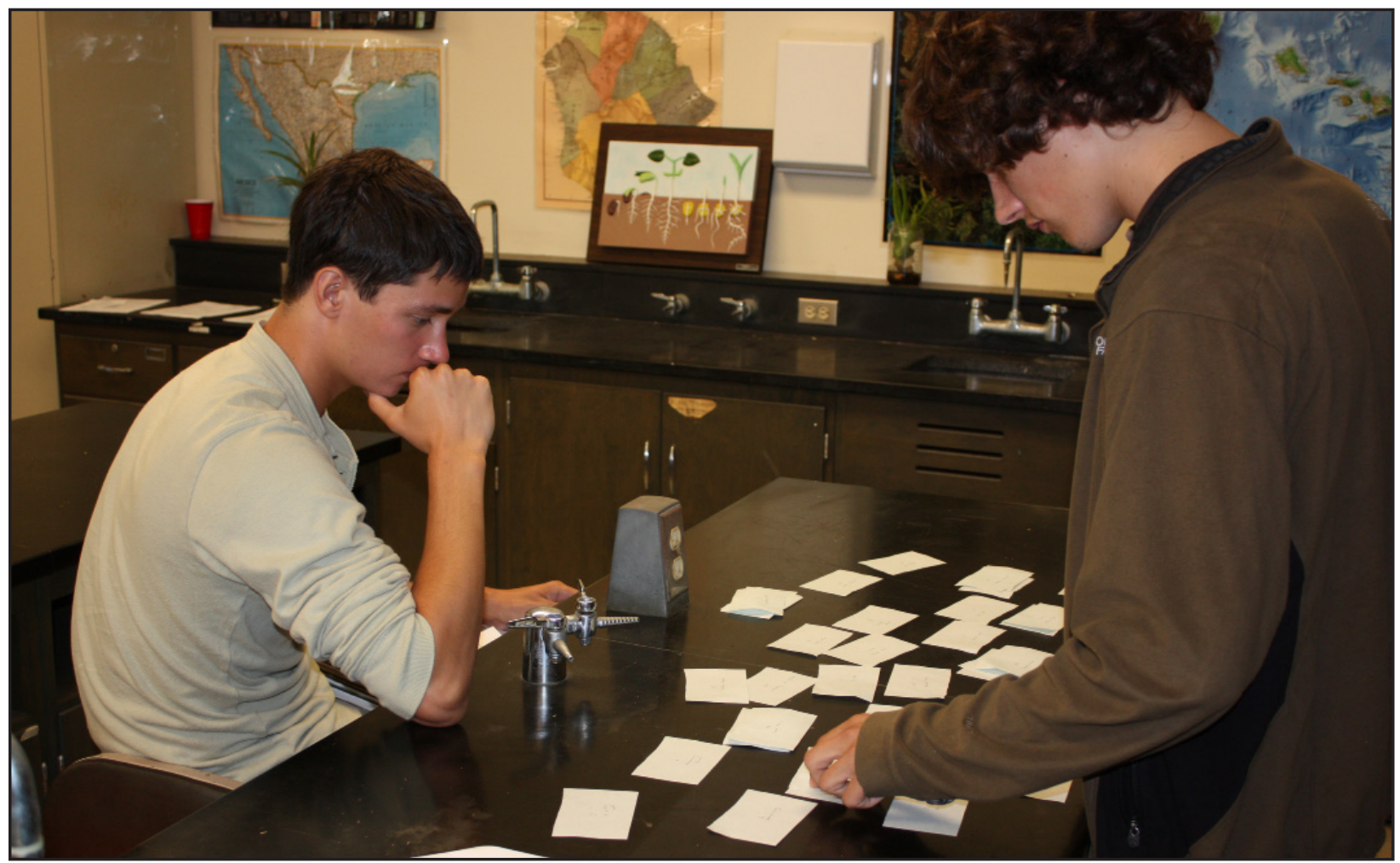

Figura 13. Uno studente è assorto mentre i suoi partner smistano i nomi delle piante in categorie.

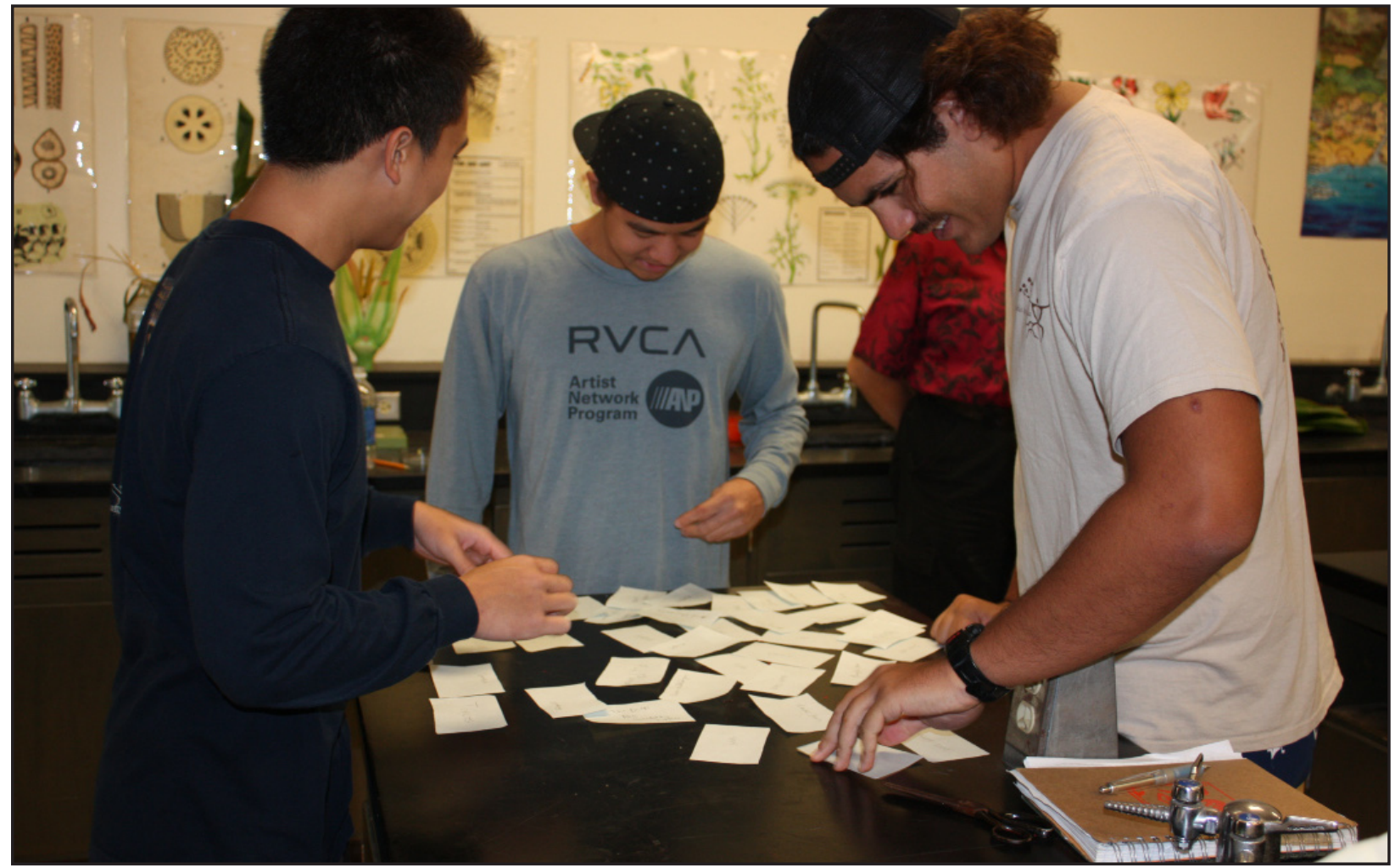

Figura 14. Differenti tipi di comportamenti sociali, come ad esempio discussioni collaborative, sono stati osservati durante l'esercizio di categorizzazione. 


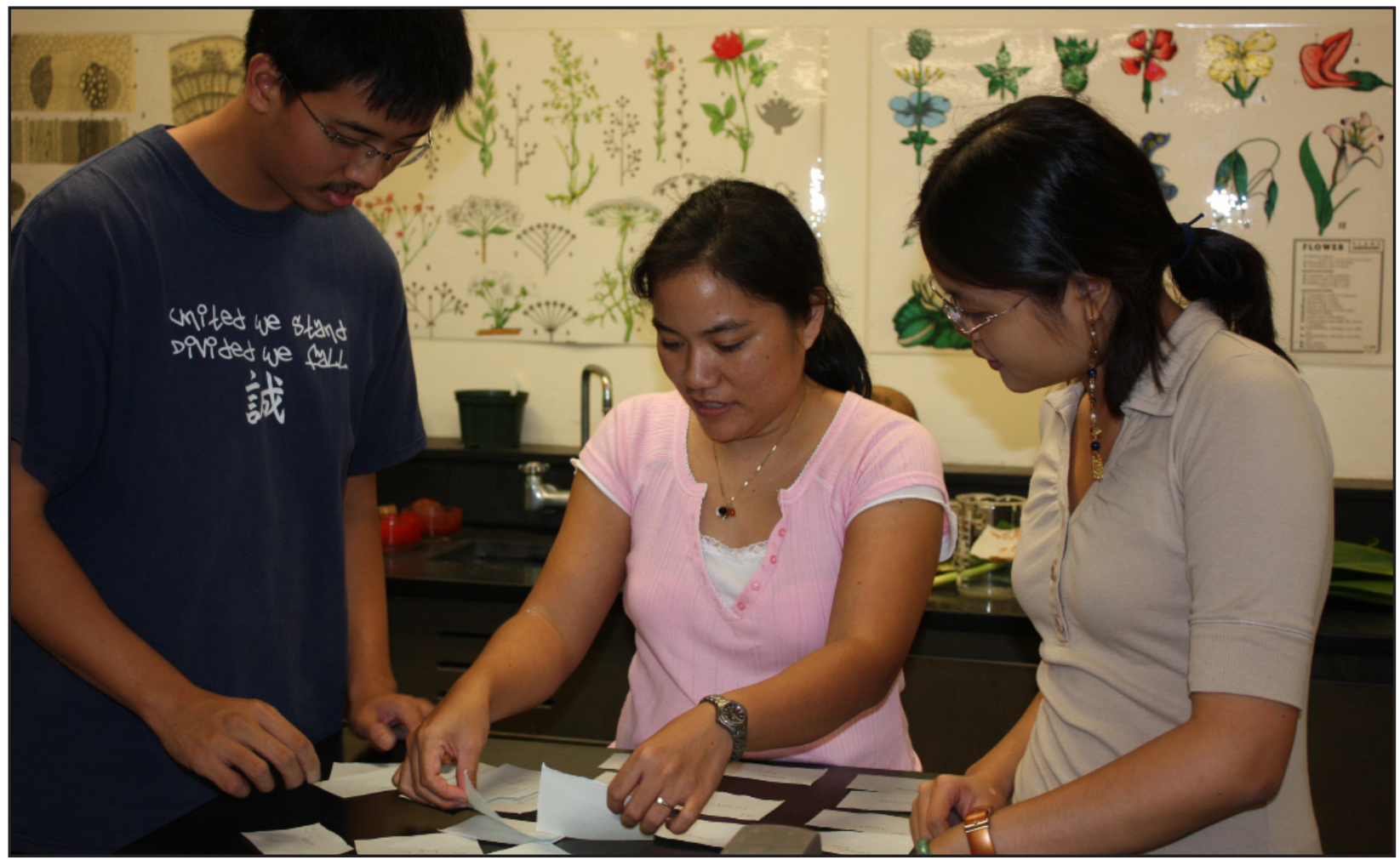

Figura 15. Un certo comportamento di leadership è talvolta emerso durante la fase di categorizzazione dei cartoncini.

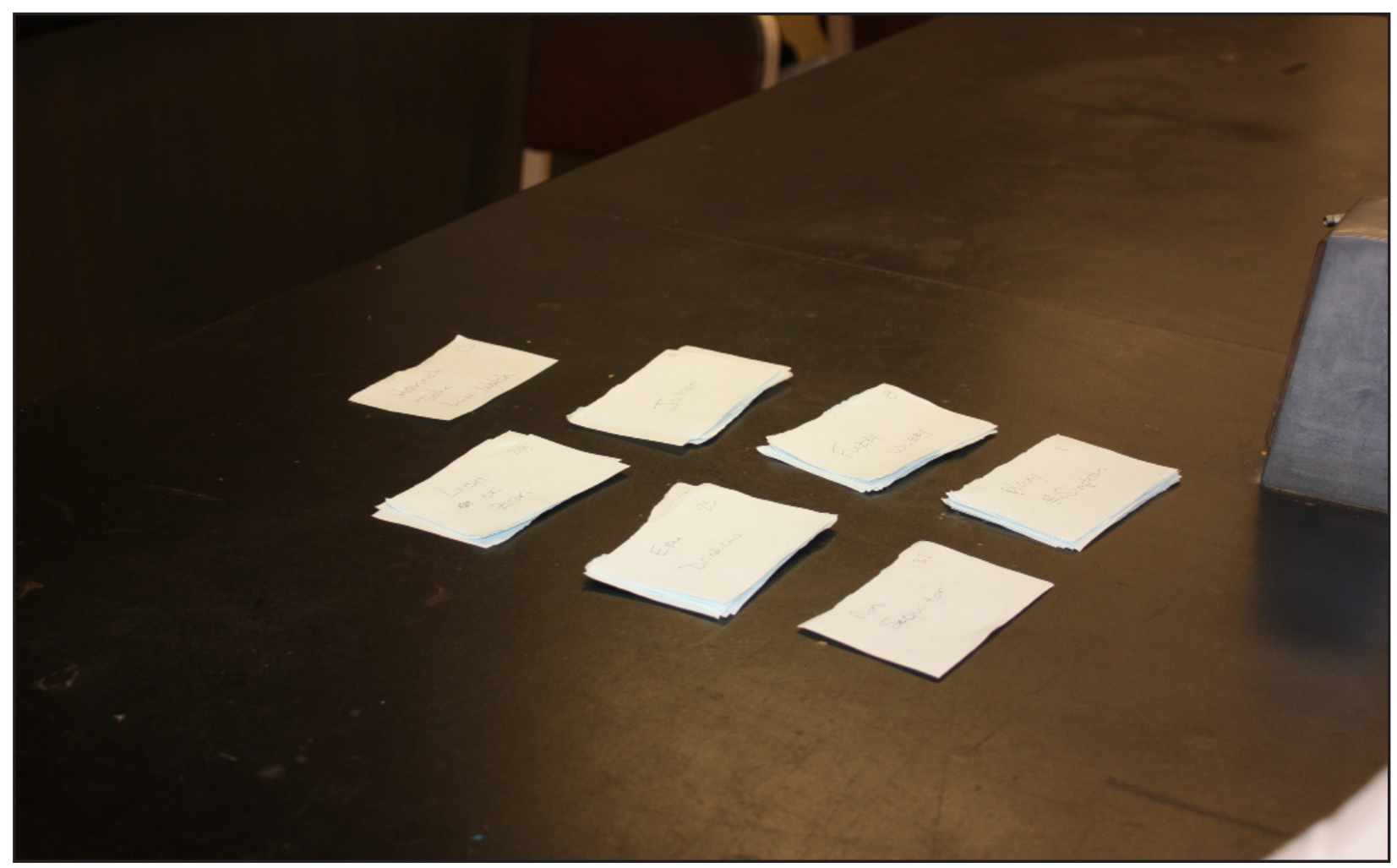

Figura 16. Un esercizio di categorizzazione dei cartoncini completato dove ad ogni pila corrisponde una categoria. 


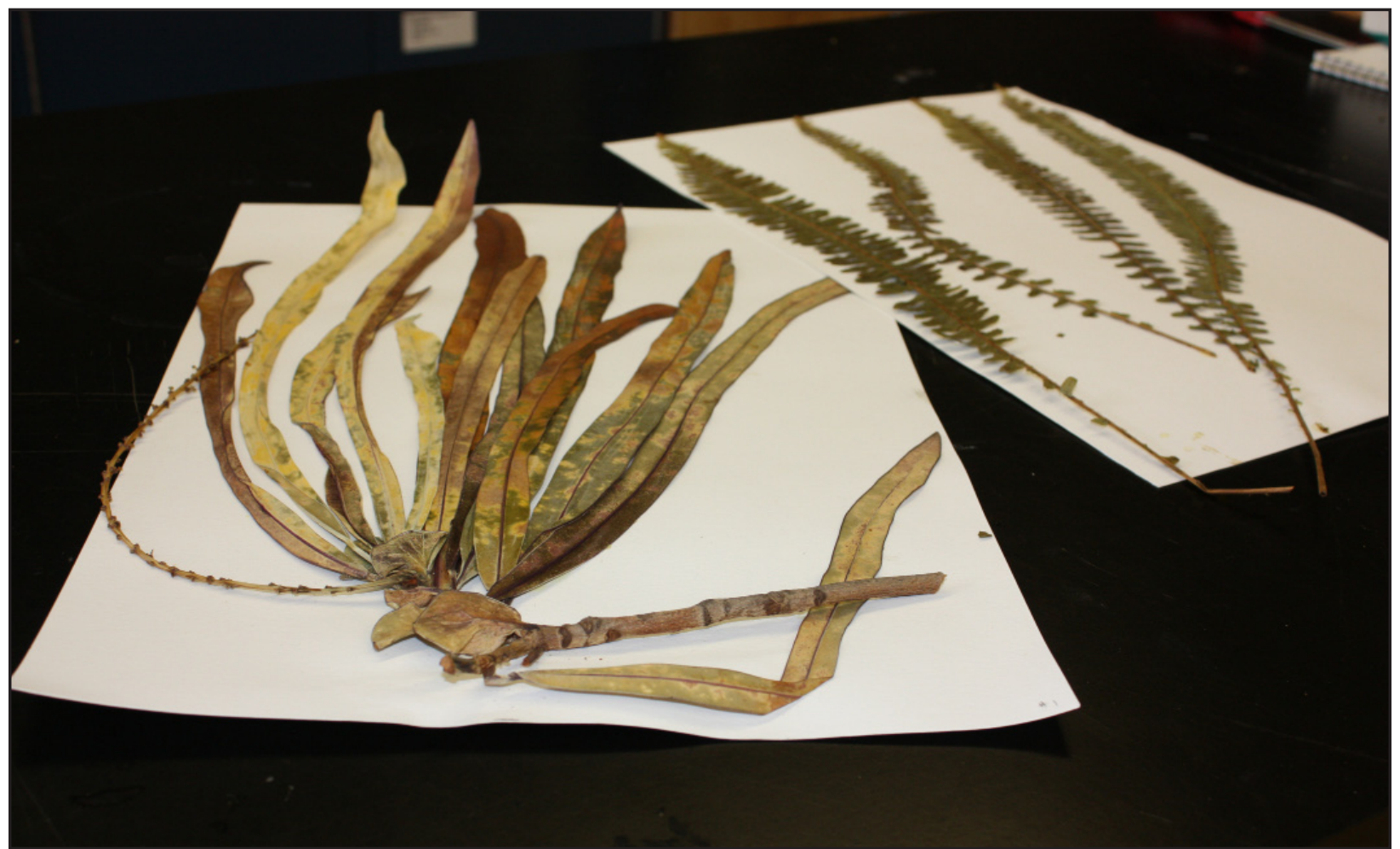

Figura 17. Una pianta che è stata allestita come campione d'erbario per essere depositata all'University of Hawai'i Herbarium (HAW).

\section{Conclusioni}

Queste immagini fotografiche non sono solo esplicative di un esperimento didattico etnobotanico, ma sono utili anche per evidenziare reazioni umane come curiosità ed interesse in un esercizio botanico creativo. La creatività degli studenti è stata stimolata mediante i processi di nominare e categorizzare le piante con cui questi hanno potuto sia vivere l'esperienza di evidenziare caratteristiche distintive e peculiari nel dare i nomi e, al contrario, cercare le similarità durante il processo di categorizzazione. In certe circostanze è stato utilizzato un sistema binomiale con sostantivo e aggettivo. Questo sistema è stato particolarmente evidente nei casi ove siano state utilizzate parole hawaiane in quanto la grammatica hawaiana prevede che una lista di sostantivi sia seguita dall'aggettivo qualificante. Questa esperienza può essere utile per capire i concetti di nomenclatura scientifica e di specie e la tassonomia popolare. Una cosa importante da sottolineare è che in questo esercizio le piante sono state selezionate in modo da includere un ampio range di proprietà nelle forme, colori e al tatto). Per questo raccomandiamo che i ricercatori che sono interessati a condurre lo stesso esperimento usino le stesse specie vegetali da noi utilizzate. In caso una particolare specie non sia disponibile, un'altre specie con caratteristiche morfologiche simili può essere utilizzata come sostituta. Infine, una possibile variazione di questo esperimento potrebbe essere quello di chiedere agli studenti di identificare le piante in base al sistema di nomenclatura che sia risultato il più utilizzato dopo che l'esperimento sia stato ripetuto per diversi anni. Questo potrebbe supportare ulteriormente l'ipotesi che gli studenti possano mostrare simili sistemi di pensiero.

\section{Ringraziamenti}

Si ringraziano gli studenti del 2008 e gli assistenti del 2007 e 2008 (Anthony Amend, Katie Kamelamela, Tamara Wong e Laura Weiss) del corso autunnale di "Introductory Ethnobotany" tenutosi all' University of Hawai i at Manoa per la loro partecipazione a questo progetto.

\section{Letteratura citata}

Berlin, B. 1973. Folk Systematics in Relation to Biological Classification and Nomenclature. Annual Review of Ecology and Systematics 4: 259-271.

Berlin, B., D.E. Breedlove \& P.H Raven. 1966. Folk Taxonomies and Biological Classification. Science 154(3746): 273-275.

Bernard, H.R. 2000. Social Research Methods: Qualitative and quantitative methods. Sage Publication Inc., Thousand Oaks, California. 
Ceuterick, M. \& P. Van Damme. 2006. Indigenous use, nomenclature and classification of plants in a Nahuatlspeaking village in the Balsas- Basin, Guerrero, Mexico. Pp. 533-536 in Ethnobotany: At the Junction of the Continents and the Disciplines. Edited by Ertug F. Proceedings of the Fourth International Congress of Ethnobotany (ICEB 2005). Istanbul, Ege Yayinlari.

Lau, Y.H., W.C. McClatchey, D. Reedy, A.K. Chock, K.W. Bridges, \& Z. Ritchey. 2009. Are Our Students Taxonomically Challenged or Not? Ethnobotany Research \& Applications 7:29-37.

McClatchey, W.C., L.X. Gollin \& S.R. Rakotonandrasana. 2005. Ethnobotanical Reseach at Analalava, Madagas- car: A Photo Essay. Ethnobotany Research \& Applications 3:391-403.

Reedy, D. Ethnobotany Segue to Science. Ethnobotany Research \& Applications, in review.

Sinclair, R.C., A.S. Soldat, \& M.M. Mark. 1998. Affective cues and processing strategy: Color coded forms influence performance. Teaching of Psychology 25:130-132.

Vogl-Lukasser, B. \& C.R. Vogl. 2005. Ethnobotanical Research in Homegardens of Small Farmers in the Alpine Region of Osttirol (Austria): Photo Essay. Ethnobotany Research \& Applications 3:79-97. 